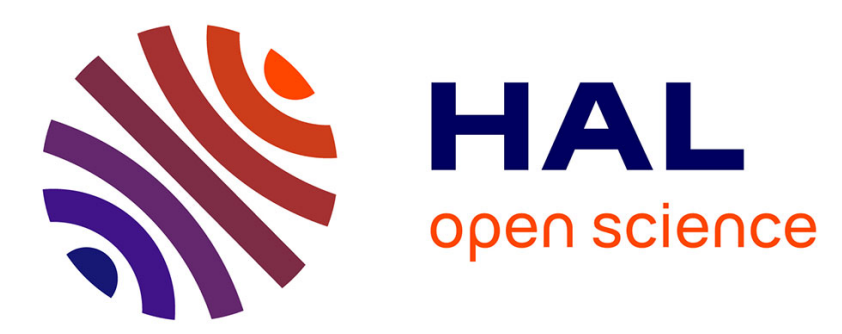

\title{
Large mobility of dry snow avalanches: insights from small-scale laboratory tests on granular avalanches of bidisperse materials
}

\author{
F. Moro, Thierry Faug, H. Bellot, F. Ousset
}

\section{- To cite this version:}

F. Moro, Thierry Faug, H. Bellot, F. Ousset. Large mobility of dry snow avalanches: insights from small-scale laboratory tests on granular avalanches of bidisperse materials. Cold Regions Science and Technology, 2010, 62 (1), p. 55 - p. 66. 10.1016/j.coldregions.2010.02.011 . hal-00489984

\section{HAL Id: hal-00489984 \\ https://hal.science/hal-00489984}

Submitted on 7 Jun 2010

HAL is a multi-disciplinary open access archive for the deposit and dissemination of scientific research documents, whether they are published or not. The documents may come from teaching and research institutions in France or abroad, or from public or private research centers.
L'archive ouverte pluridisciplinaire HAL, est destinée au dépôt et à la diffusion de documents scientifiques de niveau recherche, publiés ou non, émanant des établissements d'enseignement et de recherche français ou étrangers, des laboratoires publics ou privés. 


\title{
Large mobility of dry snow avalanches: insights from small-scale laboratory tests on granular avalanches of bidisperse materials
}

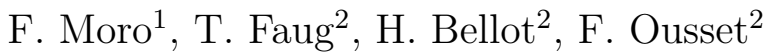 \\ ${ }^{1}$ Università degli Studi di Padova, Dipartimento di Geoscienze, I-35137, Padova, Italia, \\ ${ }^{2}$ UR ETGR, Cemagref Grenoble, BP 76, 38402 Saint-Martin d'Hères, France
}

\section{Abstract}

This paper reports small-scale laboratory tests on granular avalanches of bidisperse materials made of fine particles and larger ones. These experiments were motivated by a recent study on the rheology of dense flowing snow which provided evidence for relevant similarities in flow behavior between bidisperse granular materials and dry cold snow [Rognon and others, J. Rheol., 52, 3 (2008)]. The mass proportion of fine particles in the initial binary mixture was systematically varied at constant initial released volume, and we measured the resulting flow depth, the avalanche front velocity and the final avalanche runout. In particular, we show that the avalanche mobility is largely increased, about $40 \%$ in our tests, when the mass proportion in fine particles reaches a critical value, around 0.25 in our tests. The avalanche deposit is shallow and lengthened for this critical mass proportion in fine particles. The experimental results are interpreted by the existence of different avalanche mobility regimes on the basis on an heuristic model previously reported in literature. Finally, we discuss their possible implications for the dynamics of full-scale dry snow avalanches. 
Key words: snow, avalanche dynamics, granular flows, binary mixtures, experiments, mobility

\section{Introduction}

Snow is a cohesive granular material and granular flows are often considered to model dense avalanches of dry and cold snow (Hutter, 1996; Issler, 2003; Naaim and others, 2004). Dry flowing snow is a granular polydisperse material as corroborated by recent investigations on some deposits of full-scale snow avalanches (Bartelt and McArdell, 2009). Recent systematic experiments on a decametric snow chute has evidenced the fact that dry, cold and dense flowing snow can be seen as a mixture of snow grains, with a diameter typically smaller than $1 \mathrm{~mm}$, and of larger snow aggregates with a maximum size close to the flow thickness (Rognon and others, 2008). These experiments have been combined with discrete element simulations of bidisperse granular materials (Rognon and others, 2007). It has been shown that dry, cold and dense flowing snow exhibits a behavior steming from specific properties of bidisperse granular flows (Rognon and others, 2008). First, Rognon and others (2008) showed that steady and uniform flows are possible in a range of slope between two limit angles $\theta_{\min }$ and $\theta_{\max }$. Furthermore, when approaching the critical angle $\theta_{\min }$, the velocity abruptly drops to zero. These features are reminiscent of the behavior of granulars materials (GDR-MiDi, 2004; Forterre and Pouliquen, 2008). Second, velocity profiles measured by Rognon and others (2008) suggest that the snow flow is divided into two layers: a highly sheared basal layer of small thickness and made of snow grains is surmounted by a thick and less sheared layer made of snow 
aggregates. It corresponds to a behavior close to the behavior of bidisperse granular mixtures (Rognon and others, 2007). These recent results suggest that assimilating dry, cold and dense flowing snow to a bidisperse granular fluid is a rather good approximation. Many previous small-scale laboratory tests to model snow avalanches have been carried out using monodisperse granular flows to predict avalanche trajectories (Savage, 1979; Savage and Hutter, 1989) or, more recently, to study the influence of obstacles (Tai and others, 2001; Hákonardóttir and others, 2003; Faug and others, 2004, 2008). In this paper we investigate experimentally flows of bidisperse granular mixtures in order to quantify the influence of the bidispersity on the avalanche dynamics. The thickness and the flow velocity during the propagation phase, as well as the maximum runout distance and the final shape of the deposit in the runout zone, are studied here. Our results show that these macroscopic flow descriptors, of crucial interest for hazard zoning and risk management, are largely influenced by the bidispersity. In particular, it exists a mass proportion in fine particles for which the runout distance is maximum. This result demonstrates that bidispersity may be a good candidate to contribute to enhance the mobility of granular snow avalanches, and more generally, of geophysical granular flows.

First, we describe the experimental set-up and procedure with a focus on an advanced experimental technique to catch the shape of the granular mass. The second part presents the results concerning the influence of the binary mixture composition on (i) the granular deposit in the runout zone and (ii) the flow characteristics in the upstream flume. The main result is the observation of an enhanced mobility at a given mass proportion in fine parti- 
cles inside the granular mixture. Then, we try to highlight various mobility regimes depending on the mass proportion in fine particles, on the basis of an heuristic model recently proposed by Philipps and others (2006). Finally, the results and their possible implications for snow avalanche dynamics are discussed.

\section{Experimental device and procedure}

The experimental device we used corresponds to a geometry typically used to study snow avalanches on reduced models (Hákonardóttir and others, 2003; Faug and others, 2003, 2008), and more generally geophysical flows (Hutter and others, 1995). It consists of a system of two inclined planes including: (i) a reservoir to store the material and equipped with a gate to release it, (ii) a steep inclined plane equipped with a flume inside which the granular material propagates, (iii) a downstream plane inclined at a lower slope angle where the granular mass decelerates, spreads and finally stops. A typical granular deposit is thus generated. The slope angle of the flume corresponding to the propagation zone was fixed at $35^{\circ}$ and the one corresponding to the runout zone was fixed at $6^{\circ}$ for the whole experiments. Fig. 1 shows a sketch of the experimental device with the associated instrumentation.

As already mentioned above, most of the previous studies available in literature deal with monodisperse granular flows. Some recent studies tackle the issue of bidisperse granular flows and the associated enhanced mobility using 2D (channelized flows) small-scale laboratory tests (Phillips and others, 2006) or 2D discrete numerical simulations (Linares-Guerrero and others, 2007). Philipps and others (2006) performed experiments with granular sys- 
tems made of rectangular (rafter with typical size $75 \mathrm{~mm} \times 25 \mathrm{~mm} \times 2.2$ $\mathrm{mm}$ ) or spherical (gravel of diameter 10-20 mm) coarse particles mixed with fine spherical particles (about $100 \mu \mathrm{m}$ in size). Their bimodal granular systems are characterized by a few number of coarse (rafter or gravel) particles. In this paper, we analyse 3D granular avalanches involving a great number of fine and large spherical particles using advanced experimental techniques. It gives us access to a large number of measures to characterize the 3D (lateral extension) avalanche deposit: maximum length, location of the center of mass, spreading length above the center of mass, mean width, flow depth profile of the deposit, location of the maximum depth in the main flow direction. Thus, the tests presented here are full 3D flows and the observed enhanced mobility can be characterized by additional parameters that were not studied in (Phillips and others, 2006; Linares-Guerrero and others, 2007). The bidisperse granular materials consists of glass beads of diameter $d_{2}=1 \mathrm{~mm}$ mixed homogeneously (before releasing the whole granular mass) with glass beads of diameter $d_{1}=200 \mu \mathrm{m}$. The value $d_{1}$ is a mean value: a slight polydispersity characterizes the fine particles whose diameter ranges from 150 to $250 \mu \mathrm{m}$. The ratio $D=d_{2} / d_{1}$ is then between 4 and 6.66 being a value sufficiently large for the occurrence of segregation processes. Indeed, it is known that segregation starts to occur above $20 \%$ in polydispersity (Hirshfeld and Rapaport, 1997; Berton and others, 2003) which corresponds to a ratio $D=d_{2} / d_{1}$ typically higher than 1.2. The mass initially released was kept constant and equal to $m=10 \mathrm{~kg}$. The channel roughness was smooth (wooden surface) relatively to the roughness of the runout zone made of sandpaper glued on the inclined plane. We will come back to this point when giving an attempt 
to analyse the typical values of the ratios $d_{1} / \lambda_{r}$ and $d_{2} / \lambda_{r}$ with regard to the recent results on bidisperse granular media (Goujon and others, 2003, 2007), $\lambda_{r}$ being the typical size of the roughness asperities (sandpaper). Finally the only initial varying parameter in the experiments is the mass proportion, called $r$, in fine particles of diameter $d_{1}$ :

$$
r=\frac{m_{1}}{m}=\frac{m_{1}}{m_{1}+m_{2}},
$$

where $m_{1}$ is the mass of fine particles and $m_{2}$ is the mass of larger particles. We carried out many laboratory tests by varying the ratio $r$ between the value 0 (glass beads of diameter $d_{2}$ only) and 1 (glass beads of diameter $d_{1}$ only).

A laser sheet which generates a line parallel to the channel walls and centered on this latter (see the red line in Fig. 1) is projected on the inclined plane. When the granular mass propagates on the channel, this laser line is deflected. Thanks to a video camera placed normal to the channel bottom, we can visualize the shifted laser line (video camera $n^{\circ} 1$ on Fig. 1). The deviation is directly proportional to the flow thickness. A calibration phase using various rectangular objects of known thickness allows us to determine the proportionality coefficient. Then, the thickness at each time $t$ and at each point $X$ (in the centerline of the channel) can be easily derived. Fig. 2 gives a depth-profile in time obtained at various abscissa of the channel in the investigation zone of the laser sheet. This latter is filmed by the video camera $\mathrm{n}^{\circ} 1$, has a length of $1 \mathrm{~m}$ and is defined on Fig. 1. Let us note that the zone inside which we measured flow thicknesses is smaller than $1 \mathrm{~m}$. The order of magnitude is $55 \mathrm{~cm}$, which corresponds to the zone between the 
locations $X_{1}$ and $X_{5}$ defined on Fig. 1. In this investigated zone and at a given location $X$, the typical depth-profile in time presents a maximum with a plateau more or less pronounced. We observe that the value of this maximum varies with the location $X$ in the channel but this variation is very weak in the ending part of the channel. The typical values of gradients of maximum flow-depth in the ending part of the channel are around $1 \mathrm{~mm}$ (see Fig. 2). The precision of depth measurements has been estimated to $1 \mathrm{~mm}$. We systematically defined $h$ as the mean value of the three maxima obtained at locations $X_{3}, X_{4}$ and $X_{5}$, in the bottom part of the channel, excluding the maxima obtained at locations $X_{1}$ and $X_{2}$ in the top part. Locations $X_{i=1,2,3,4,5}$ are defined on Fig. 1 . It is then possible to analyse the influence of the ratio $r$ on the flow-depth $h$. These results are presented and discussed in the following section.

The laser line allows us also to determine the locations of the front $x_{f}$ in the channel and, consequently, the propagation speed of the granular front (Lagrangian front speed), in want of the knowledge of the depth averaged flow speed of the granular avalanche core at a given location $X$ (Eulerian speed). The point corresponding to the front position is the point downstream which the laser line is not shifted and upstream which it is shifted. Fig. 3 gives an example of the front position $x_{f}$ versus the time. This graph allows us to calculate the front velocity, called $u$, and defined by the slope of the straight line $\left(u=d x_{f} / d t\right)$. The precision on front speed was estimated to $0.1 \mathrm{~m} \mathrm{~s}^{-1}$. The results about the influence of the ratio $r$ on the front velocity $u$ are presented and discussed in the following section.

Parallel fringes, alternatively black and white, were projected on the gran- 
ular mass on the runout zone with the help of a projector. Similarly to the shifted laser line technique, the fringes network is shifted by the granular mass and the deviation is proportional to the deposit thickness. A video camera placed normal to the inclined plane (video camera $n^{\circ} 2$ on Fig. 1) allows us to visualize the shifted fringes network (Fig. 4b). The calculation consists in determining with accuracy the phase difference $\Delta \varphi(x, y)$ between the reference signal, without the granular mass, and the object signal, in presence of the granular mass which distorts the fringes. The reference signal can be modelled by the following sinusoidal signal:

$$
I_{r e f}(x, y)=\bar{I}(x, y)+\bar{I}(x, y) \gamma(x, y) \cos \left(\varphi_{0}+2 \pi f_{0} x\right),
$$

where $\bar{I}(x, y)$ is the mean intensity of the signal, $\gamma(x, y)$ the lightness contrast at the point location $(x, y), f_{0}$ is the frequency linked to interfringe and $\varphi_{0}$ the origin phase. The object signal can be modelled by the following sinusoidal signal:

$$
I_{\text {object }}(x, y)=\bar{I}(x, y)+\bar{I}(x, y) \gamma(x, y) \cos \left(\varphi_{0}+2 \pi f_{0} x+\Delta \varphi(x, y)\right) \text {, }
$$

The phase difference $\Delta \varphi(x, y)$ between the two signals is directly proportional to the local deviation of fringes $\delta(x, y)$ :

$$
\Delta \varphi(x, y)=2 \pi f_{0} \delta(x, y)
$$

It is possible to derive theoretically the proportionality coefficient relating the local deviation of the fringes and the object height. For this aim, the geometrical and optical features of the system formed by the projector, the 
surface of the inclined plane and the video camera have to be considered (Sansoni and others, 1999; Vincent, 2003). However, in order to avoid errors linked to the determination of these geometrical features and to optics of instruments, we used an empirical calibration thanks to rectangular objects of known height to deduce the deposit thickness at each point $(x, y)$ of the inclined plane. This experimental technique using fringes projection is now currently used to study flows in small-scale laboratory models (Pouliquen and Forterre, 2002; Cochard and Ancey, 2008).

Several methods exist to have access to the phase difference (Surrel, 2000; Vincent, 2003). Some methods are based on a single image projection of the fringes network as the Fourier transform method (global approach). Other methods, more accurate, are based on the principle of phase shifting which consists in projecting several images shifted one to each other (local approach). This second approach is not adapted to dynamic problems with rapid changes in time but it brings an increased precision for static (or quasistatic) problems. In the experiments presented here, we use this second approach by projecting four grids shifted of $\pi / 2$. The displacement of fringes by the granular mass is interpreted as a fringes phase modulation and can be expressed according to the four intensities $I_{i}(x)$ :

$$
\phi(x)=\arctan \left(\frac{I_{4}(x)-I_{2}(x)}{I_{1}(x)-I_{3}(x)}\right),
$$

where the four shifted images $\left(\phi_{1}=0, \phi_{2}=\pi / 2, \phi_{3}=\pi, \phi_{4}=3 \pi / 2\right)$ are modelled by a sinusoidal signal: $I_{i}(x, y)=\bar{I}_{i}(x, y)+\bar{I}_{i}(x, y) \cos (\phi(x)+$ $\left.\Delta \phi_{i}(x)\right)$. The thickness is then directly proportional to the phase modulation $\phi(x)$ and is obtained by calibration. The calibration procedure consists in 
projecting the four reference shifted images $\left(\phi_{1}=0, \phi_{2}=\pi / 2, \phi_{3}=\pi\right.$, $\left.\phi_{4}=3 \pi / 2\right)$ on various rectangular objects of known height. Fig. 4 shows the projected fringes on a deposit as well as the numerical result for the deposit shape in three dimensions. The precision varies according to the image quality and is generally smaller than $500 \mu \mathrm{m}$.

Experimental tests consisted in releasing a mass of $10 \mathrm{~kg}$ and changing the ratio $r$ at each test. We tested values of $r$ ranging from to 0 to $65 \%$ with a $1 \%$ step. For values greater than $65 \%$, we did tests with the following values: $80 \%$ and $100 \%$. In the following section, we present results concerning the influence of the ratio $r$ on (i) the deposit morphology and (ii) the flow dynamics inside the ending part of the flume.

\section{Results}

\subsection{Evidence of segregation}

The qualitative observation of deposits, as displayed in Figs. 5a and 5b, shows (i) the presence of fine particles at the base of the deposits, and (ii) the presence of coarse particles mostly at the front and, to a less extent, at the sides. These observations, previously discussed by Pudasaini and Hutter (2007, p. 63, figure 2.6), are the proof of size segregation effects, during the propagation phase and during the stopping phase, where the fine particles tend to fall (percolation) and large grains tend to go toward the surface.

The present paper does not tackle the strickly speaking segregation process, largely studied for relatively dense granular flows (Savage and Lun, 1988; Ottino and Khakhar, 2000; Félix and Thomas, 2004; Gray and Chugunov, 2006) but it rather analyses the consequences of the segregation process on 
the granular avalanche, during its propagation and stopping phases. Our experiments show, which is described in detail in this section, that several features of the granular avalanche are influenced by the ratio $r$ : (i) the velocity and the thickness during the accelerating propagation phase, (ii) the final length of the deposit in the runout zone, (iii) the thickness of the final deposit (maximum value and mean value along the main flow direction in the $x$-axis direction), (iv) the location where the deposit thickness is maximum.

\subsection{Deposit in the runout zone}

\subsubsection{Deposit features influenced by the binary mixture composition}

Fig. 6 shows various features of the granular avalanche deposit versus the mass proportion in fine particles $r$ : length $L$, position $x_{G}$ of the center of mass, spreading length $x_{\text {spreading }}$ above the center of mass, maximum width $\ell$, maximum height $H_{\max }$, mean depth along the centerline of the deposit $H_{x}$ and position $x\left[H_{m a x}\right]$ of the maximum height. Each dimension $x$ has been normalized by the corresponding dimension, $x_{2}$, obtained with the monodisperse mixture of beads with diameter $d_{2}=1 \mathrm{~mm}$. In the following, the results are systematically normalized by the values obtained with the monodisperse mixture made of beads with diameter $d_{2}=1 \mathrm{~mm}$ (reference values with subscript 2). In Fig. 6, for the dimensions $L$ and $\ell$, are given the results obtained from the fringes projection technique combined with phase shifting as well as the results from the direct measure with a ruler. Similar results are obtained with both methods, which validates our implemention of the fringes projection technique.

The deposit length is very sensitive to the ratio $r$. It displays a maximum around $r=0.2-0.3$ and this maximum reaches the value 1.4. It shows 
that the runout distance with a bidisperse mixture (between 20 and 30\% in mass of fine particles) is increased of nearly $40 \%$ in comparison with a monodisperse mixture of large particles. Let us note that $L / L_{2}$ is slightly smaller than 1 when $r=1$, which illustrates that the runout with fine particles only is slightly shorter than the runout with large grains only. This observation shows that large particles go further than small ones, for fixed roughness conditions. We can introduce here the critical diameter $d_{c}$ defined by Goujon and others (2007), and which depends on the typical size $\lambda_{r}$ of the downstream plane roughness and its compacity $C$ :

$$
d_{C}=\frac{\pi}{6 C \sqrt{3}} \lambda_{r} \approx 0.3 \frac{\lambda_{r}}{C}
$$

For monodisperse dry granular flows down inclined planes, there exist a diameter $d_{C}$ of particles for which the basal friction is maximum: minimum in mean velocity, maximum in deposit thickness and minimum in deposit length (Goujon and others, 2003). The value of $d_{c}$ is equal to $0.42 \lambda_{r}$ for a typical roughness compacity of 0.72 , mean value between the maximum compacities in two dimensions $\left(C_{2 D} \approx 0.64\right)$ and three dimensions $\left(C_{3 D} \approx\right.$ 0.8). The mean value of $\lambda_{r}$ is $250 \mu \mathrm{m}$ (corresponding to the norm FEPA for the sandpaper P60 used in our tests), which gives the value $d_{c} \sim 105$ $\mu \mathrm{m}$. The diameter $d_{2}$ is nearly ten times greater than the diameter $d_{c}$, which implies that the $1 \mathrm{~mm}$ in diameter beads can not be trapped easily in the roughness $\left(d_{2} / \lambda_{r} \sim 4\right)$. On the other hand, the diameter $d_{1}$ is less than twice greater than $d_{c}\left(d_{1} / \lambda_{r} \sim 0.8\right)$, which implies that fine particles of $200 \mu \mathrm{m}$ in diameter are more easily trapped. It results, at constant initial mass and slope angles, a runout greater for the large grains of diameter $d_{2}$. 
However the difference between the two monodisperse deposits is not so large. We may think it is caused by the very low value of the runout zone slope angle $\left(6^{\circ}\right)$ in our experiments, which allows the large grains to come at rest. Goujon and others (2007) investigated flows at higher slope angles on which grains were less stable and they obtained a larger difference between the two monodisperse deposits.

Thanks to the fringes projection technique, we could determine with accuracy the position of the center of mass $x_{G}$ (calculated from the beginning of the runout zone: $x=0$ in Fig. 1). $x_{G} / x_{G 2}$ (Fig. 6) displays a more pronounced maximum (value around 1.5) than $L / L_{2}$. This implies that the center of mass is more sensitive than the deposit front to the proportion in fine particles. Let us consider a simple model for which the total maximum runout is the sum of the position of the center of mass $\left(x_{G}\right)$ and a spreading length above the center of mass $\left(x_{\text {spreading }}\right)$, as previously suggested in (Faug and others, 2004):

$$
L=x_{G}+x_{\text {spreading }}
$$

A similar approach was been recently proposed in (Staron and Lajeunesse, 2009) where the runout of a gravity-driven granular flow is decomposed in two contributions: a so-called sliding along the topography and a so-called spreading of the unconsolidated mass. From this simple model, we can conclude that the segregation process acts differently on the displacement of the total mass (sliding) and on the way the unconsolidated mass spreads beyond the center of mass. Indeed, the spreading length is less sensitive to the ratio $r$ than the position of the center of mass, as shown by Fig. 6 . 
In Fig. 6 is also shown the maximum width being relatively independent of the ratio $r$. This result tends to show that the segregation acts mainly in the prevailing direction of the avalanche propagation (simple shear flow). A similar effect has been also observed by Zanuttigh and Di Paolo (2006).

The fringes projection technique allows us to study other effects on the deposit shape: the mean depth-profile, $H$, and the maximum height, $H_{\max }$, as well as the point location where the height is maximum, $x\left[H_{\max }\right]$. The height $H$ is defined by:

$$
H=\frac{S}{\ell_{S}}
$$

where $S$ is the area of the transverse section of the deposit at a given abscissa $x$ and $\ell_{S}$ is the deposit width at the abscissa $x$. The height $H$ corresponds to the mean value in the transverse $y$-axis direction (Fig. 1). Fig. 7 shows examples of mean depth-profiles obtained from various values of the ratio $r$. The segregation is shown to decrease the thickness of the deposits. At the critical value of the ratio $r$ (around $0.2-0.3$ ), the deposits are lengthened and thinner. At values greater and smaller than the critical ratio, the deposits are thicker and shorter.

Fig. 6 also shows the maximum deposit height, $H_{\max }$, as well as the mean depth along the centerline of the deposit, called $H_{x}$. Both $H_{\max }$ and $H_{x}$ are very sensitive to $r$. The height reduction is maximum (around 35-40\%) at the critical ratio $r_{\text {crit }}=0.2-0.3$.

If we neglect the changes in volume fraction (we will come back to this point later in the paper), we can assume that the volume is constant because we kept the total mass $m$ constant. As the deposit width is independent of 
the ratio $r$, it results that the average depth along the whole profile, $H_{x}$, is, in first approximation, inversely proportional to the deposit length $L$. This result is displayed in insert in Fig. 8.

In Fig. 6 is finally displayed the position of the maximum height, $x\left[H_{\max }\right]$. The results are more scattered because this parameter is more sensitive to experimental conditions. However, the curve (see the gray trend line reported in Fig. 6) shows that this parameter displays a behavior similar to the one observed for $L$ and $x_{G}$. Let us remark that the maximum is very pronounced since the value of $x\left[H_{\max }\right]$ is nearly double when $r=r_{\text {crit }}$, in comparison with large particles only. This result indicates that the deposit morphology is very sensitive to $r$, and therefore to segregation effects.

\subsubsection{Existence of a critical proportion in fine particles for which the friction} is minimum?

Rognon and others (2007) studied, on the basis of discrete numerical simulations, velocity profiles of 2D bidisperse steady flows of disks with varying the ratio $S_{g}$ as well as the size ratio $D=d_{2} / d_{1}$. $S_{g}$ was defined as the surface proportion of large disks (Rognon and others, 2007): $S_{g}=\left(n_{2} d_{2}^{2}\right) /\left(n_{2} d_{2}^{2}+n_{1} d_{1}^{2}\right)$, where $n_{2}$ is the number of large grains and $n_{1}$ the number of small grains. They observed an increase in thickness of the top layer made of large grains when increasing $S_{g}$, and for $S_{g}=3 / 4$, the basal layer of fine grains was shown to disappear. By analogy, we can define the dimensionless number $V_{g}$ representing the volume proportion of large grains (1 $\mathrm{mm}$ glass beads): $V_{g}=\left(n_{2} d_{2}^{3}\right) /\left(n_{2} d_{2}^{3}+n_{1} d_{1}^{3}\right)$. Since large and small particles has the same density (glass beads), the volume proportion of large grains is equal to the mass ratio between the large grains and all the grains: $V_{g}=1-r$. 
According to Fig. 6, the various parameters defined previously $L / L_{2}$, $x_{G} / x_{G 2}, x_{\text {spreading }} / x_{\text {spreading } 2}, x\left[H_{\max }\right] / x\left[H_{\text {max } 2}\right]$ increase when decreasing $r$ (when increasing $\left.V_{g}\right)$ and reach a maximum when $r$ is around 0.2-0.3 $\left(V_{g}\right.$ around $0.7-0.8$ ). These parameters then decrease to tend toward 1, extreme value for which there is no more fine grains $\left(n_{1}=0\right)$. These results appear to be compatible with the observations by Rognon and others (2007). In this decreasing part of the curves, everything acts as if there was no enough fine grains to form a homogeneous basal layer allowing the optimal lubrication of the whole granular mass mainly made of large grains. In order to develop segregation processes that are able to contribute to avalanche lengthening, it is necessary to exceed a given proportion in fine particles inside the mixture. Conversely, this proportion should be not too high in order to maintain the efficiency of the segregation processes. It is really a challenge to find the critical value theoretically. However we can provide a qualitative physical explanation to the critical condition. Below $r=0.2-0.3$ (above $V_{g}=0.7-$ 0.8 ), the lubrication can act but is not optimal. The optimum is probably reached when a homogeneous layer of fine particles is formed at the base of the flow and, simultaneously, no -or a few- fine particles are present inside the voids between the large particles. Above $r=0.2-0.3$ (below $V_{g}=0.7-0.8$ ), as the basal layer of fine particles is saturated, the added fine particles start to fill the voids between the large particles. This process offers additional contact surfaces for interparticle friction (the velocity of the large particles is decreased) which counteracts the basal lubrication.

These mechasnisms can be interpreted by the existence of a critical proportion for which the friction is minimum. The observed maxima on the 
curves correspond to this minimum friction. These results are in agreement with the results front 2D discrete numerical simulations showing that the friction coefficient versus the proportion in fine particles displays a minimum (see figure 11 in (Linares-Guerrero and others, 2007)).

\subsubsection{Volume of the avalanche granular deposit}

The fringes projection technique combined with phase shifting allowed us to access to the final volume of the deposit. This volume is also a function of $r$ as shown in Fig. 8. In spite of weak variations, it illustrates that the assumption made previously on the volume fraction is not correct. Since we kept the total mass constant ( $m=10 \mathrm{~kg}$ ), we can deduce the volume fraction evolution:

$$
\phi(r)=\frac{m}{\rho_{P} V(r)} .
$$

At constant total mass, the volume fraction is inversely proportional to the volume. Given that a minimum is shown in the volume variation, the volume fraction displays a maximum. However, this results is non trivial to interpret since the initial volume is not perfectly controlled. Rearrangement mechanisms, and consequent variations in volume fraction, may have taken place before the avalanche release, when setting the granular mixture up inside the reservoir. Indeed, it is known that the compacity of a bidisperse granular in a static state depends on the proportion of large grains in the mixture (Ben Aim, 1970; Dodds, 1980). Adding particles of different size (larger or smaller), even in small amount, to a monodisperse medium, modifies and generally increases the solid fraction when the size ratio $D$ is sufficiently large. This increase in compacity is explained by two main 
mechanisms (Ben Aim, 1970): (i) the insertion mechanism for which small particles fill the voids inside a granular skeleton mainly made of large grains (small $r$ ) and (ii) the substitution mechanism for which a large grain replaces a cluster of fine grains and the openings between these fine grains inside a matrix mainly made of fine grains (large $r$ ). The mean volume fraction of the deposits depends strongly on the segregation processes during the propagation phase. It may also depend on the initial granular rearrangements and resulting initial compacities (difficult to control) of the granular mixture put in place inside the reservoir. However, the behavior observed in Fig. 8 remains difficult to interpret and cannot be explained simply by initial rearrangements. It probably suggests inherent mechanisms occurring during the flow and stopping phases which need further investigations in the future.

As a first approximation, we can relate the total volume of the deposit to its length $L$, its width $\ell$ and its mean thickness $H_{x}$ by the following equation:

$$
V=\alpha L H_{x} \ell=\frac{m}{\rho_{P} \phi(r)},
$$

where $\alpha$ is a shape coefficient linked to the deposit. The results show that $\alpha$ is constant (close to $3 / 4$ ) whatever the value of the ratio $r$. This result is in agreement with the observations showing, for each test, a mean shape of the deposit similar to the one given in Fig. 4a. Let us note that all the deposits observed during our tests, whatever the $r$ value, are uniforms (see Fig. 5a) in the sense that we do not observe digitation mechanism, similarly to the fingering features analysed by other authors (Pouliquen and others, 1997; Pouliquen and Vallance, 1999), and which can be also observed for some natural snow avalanches. 
In this section, we showed the influence of the segregation process on the characteristics of the final deposit. We also observed important effects in the propagation zone on the flow depth and velocity. This tends to show that segregation processes can occur at very short times once the avalanche has been initiated. We discuss this question in the following section.

\subsection{Flow in the channel}

We remind here that the channel is smooth (wooden bottom), which implies that the typical size, $\lambda_{c}$, of the channel roughness is very small in comparison with the diameter $d_{2}\left(\lambda \ll d_{2}\right)$ and also $d_{1}\left(\lambda \ll d_{1}\right)$. Contrary to the runout plane, complex effects due to coupling between the roughness and the flowing binary mixture (Goujon and others, 2003, 2007) have not to be considered for the propagation phase in the channel. Fig. 9 shows the mean flow depth $h$ (defined in section 2 ) in the channel versus the ratio $r$. The flow depth is relatively constant when the mass proportion in fine particles is weak. It decreases afterwards when the ratio $r$ reaches a critical value around $0.2-0.3$. Then, the flow depth remains relatively constant and, finally, slightly increases at high $r$ values.

In Fig. 9 is also shown the mean front velocity in the channel (defined in section 2) versus the ratio $r$. The speed increases when the mass proportion in fine particles increases, before reaching a plateau when the ratio $r$ reaches a critical value. The transition is observed around $0.2-0.3$, which corresponds to a order of magnitude similar to the value obtained for $h$. Let us note that, in spite of the weak number of available data, the speed tends to decrease at high $r$ values.

The Froude number of the free-surface flows is often considered as an 
important similitude criterion which is rather easy to estimate with a prior knowledge of the flow depth $h$ and the velocity $u$. The Froude number is defined as:

$$
F r=\frac{u}{\sqrt{g h}} .
$$

We reported the value of the Froude number versus the ratio $r$ in Fig. 9. The Froude number ranges from 5 to 9 in our tests with granular materials. It corresponds to typical values for rapid snow avalanches (Issler, 2003). The Froude number is shown to increase when increasing the mass proportion in small grains inside the mixture and it reaches a relatively constant value when the ratio is above a critical value around 0.3. Let us note that above a certain value of $r$ (around 0.6-0.7), the Froude number decreases. This observation results from the decrease in velocity and the increase in flow depth. Segregation generates thinner and more and more rapid channelized flows. These results are the proof that the segregation and the induced lubrication can be active even if the bottom surface is smooth. The trapping of fine particles is limited here. The large velocity gradients (between the basal layer of fine particles and the large particles above) needed to lubricate the flow are more due to the high inclined slope of the channel. This latter makes the large particles (high inertia) above the small moving ones $(r=$ $\left.r_{\text {crit }}\right)$ flowing down faster than the small ones alone $(r=1)$. These results are compatible with the fact that the granular avalanche covers a greater distance and the deposits are thinner and lengthened thereafter in the downstream runout zone. 


\section{Data interpretation}

On the basis of the study by Philipps and others (2006), we can interpret experimental data by distinguishing three mobility regimes identified in Fig. 10. Fig. 10 gives the deposit length, L, normalized by the deposit length, $L_{2}$, obtained for $r=0$ (large grains only) versus the mass proportion in fine particles, $r$.

The regime $(I)$ is characterized by a mixture made of a majority of large grains with a few fine particles $\left(0<r<r^{I-I I}\right.$, where $r^{I-I I}$ is the proportion corresponding to the transition toward to the regime $(I I)$ defined below). In the regime $(I)$, the fine particles, located at the base of the flow (as a consequence of segregation effects in the channel), can play the role of a rolling plane for the incoming larger grains. The limit between regimes $(I)$ and $(I I)$ is close to the maximum observed on Fig. 10. It is then taken identical to the critical value $r_{c r i t}: r^{I-I I}=r_{c r i t}=0.25$. Philipps and others (2006) proposed a similar value based on their experiments.

The regime $(I I I)$ corresponds to a mixture made of a dominant part of fine particles with a few large grains $\left(r^{I I I-I I}<r<1\right.$, where $r^{I I I-I I}$ is the proportion corresponding to the transition toward to the regime $(I I)$ defined below). As soon as the proportion in fine particles becomes dominant, their effect becomes more complex and they do not play the simple role of lubricating the mass of large grains. In the regime $(I I I)$, we expect that the interparticle friction is the main source of energy dissipation, which limits the avalanche mobility. A simple idea to model this regime is to consider that dissipations by friction are directly proportional to the surfaces involved in friction contacts (Phillips and others, 2006). Note that this simple approach 
does not take into account the dissipations due to collisions which are likely to be important inside the basal sheared layer made of fine particles with fluctuating velocities. The effect of substituting a large grain for the same mass of fine particles is to reduce the contact surfaces availables for interactions, which leads to a region weakly effective in terms of friction dissipations in comparison with regions where fine particles are free to interact. By comparing a situation with a binary mixture (mixture $A$ ) and a situation with a monodisperse mixture exclusively made of fine particles (mixture $B$ ), we can estimate the ratio $R_{f}$ between the energies dissipated by friction in each case (Phillips and others, 2006):

$$
R_{f}=\frac{n_{1} S_{1} F+n_{2} S_{2} F}{n S_{1} F}
$$

where $S_{1}=d_{1}^{2}$ (respectively $S_{2}=d_{2}^{2}$ for the large grains) is the surface of the fine particle of diameter $d_{1}$ (respectively $d_{2}$ for the large grains). The product $n_{1} S_{1} F$ is the total dissipation by friction in the mixture $B$, where $F$ is the friction force by surface unit. For the mixture $A$, at constant surface unit force $F$, the fine particles but also the large grains contribute to friction dissipations, which gives the following total dissipation: $n_{1} S_{1} F+n_{2} S_{2} F$.

Some calculations allow us to derive the ratio $R_{f}$ as a function of the diameters ratio $D$, the density ratio $\chi=\rho_{2} / \rho_{1}$ and the mass proportion in fine particles $r$ :

$$
R_{f}=\frac{1+\frac{1-r}{\chi r D}}{1+\frac{1-r}{\chi^{r} D^{3}}} .
$$

Considering that $\chi=1$ (in our experiments), and that the value of $D$ is greater than 1 , the ratio $R_{f}$ is necessarily smaller than 1 . This prediction is 
in agreement with the experimental observation showing that adding large grains in a mixture of fine particles (by keeping the total mass constant) leads to an increase in avalanche runout. We compared the prediction of this heuristic model to data on deposit length, $L$, normalized by the deposit length, $L_{2}$, for $r=0$ (large grains only). It is crucial to notice that in Fig. 10 is reported the ratio $R_{f}$ multiplied by the ratio $\left(L_{1} / L_{2}\right)$ because the data are normalized by the reference value for $r=0$. Yet, by construction, $R_{f}$ is defined with regard to a reference situation for $r=1$. We do not have many data for high $r$ values corresponding to the regime (III) but we can observe a good agreement between the data and the heuristic model. Figs. 6, 9 and 11 are relatively flat for values $r$ greater than 0.7 . Thus the limit between the regimes $(I I I)$ and $(I I)$ is $r^{I I I-I I}=0.7$ in agreement with the value proposed by Phillips and others (2006).

The regime $(I I)$ is a transition regime $\left(r^{I-I I}<r<r^{I I I-I I}\right)$ where involved mechanisms are more complex than in regimes $(I)$ and $(I I I)$. The regime $(I)$ shows that adding fine particles in a mixture of monodisperse large grains tends to lubricate the mass of large grains. This induces an increased mobility. The regime (III) shows that adding large grains to a mixture of monodisperse fine particles tends to reduce the contact surfaces avaiblable for energy dissipation by interparticle friction. This induces an increased mobility. The transition between both regimes is necessarily characterized by the existence of a maximum. In the regime $(I I)$, we observe a maximum in avalanche runout ( $L$ and $x_{G}$ show a maximum) correlated to a minimum in deposit height $\left(H_{\max }\right.$ and $H_{x}$ show a minimum). At the critical value $r_{\text {crit }}$, deposits are thin and lengthened. We reported the ratio $\epsilon^{-1}=L / H_{x}$ 
versus the mass proportion of fine particles in Fig. 11. $\epsilon^{-1}$ is defined as the ratio between the deposit length $L$ and the mean deposit height $H_{x}$. This ratio, which characterizes the deposit extension, exhibits a maximum at the critical value $r_{\text {crit }}$. We called it $\epsilon^{-1}$ to refer to the ratio $\epsilon=H_{x} / L$, classically used in depth-averaged equations models describing shallow avalanche flows $(\epsilon<<1)$. The critical value $r_{\text {crit }}$ for which the mobility is maximum is around 0.25 in our experiments and, to our knowledge, it does not exist any model to predict it. We will come back to this value in conclusion.

\section{Discussion and conclusion}

The experiments described above show the strong influence of the binary mixture composition on the averaged characteristics of the granular avalanche. Our 3D experiments are in good agreement with the experimental results about 2D, channelized, flows in (Phillips and others, 2006) and the 2D discrete numerical results in (Linares-Guerrero and others, 2007) concerning the maximum runout. They bring new results on the influence of the binary mixture composition on (i) the final shape of the full 3D deposit (maximum flow depth, the location of this latter on the main flow direction, mean width) and (ii) the dynamics of the 2D, channelized, incident flow. Apart from these results largely discussed in the paper, we close the paper by analysing the possible implications of these results on avalanches of dense and dry snow.

\subsection{Dynamics of the incoming flow and runout deposit}

We analysed the influence of the mass proportion in fine particles on the deposit in the runout zone and on the dynamics of the incoming flow. Re- 
sults show a strong influence of the mass proportion in fine particles inside the binary mixture on (i) the flow dynamics that can be characterized by the Froude number of the incoming flow $F r$ (diamond symbols in Fig. 9) and (ii) the shape of the deposit that can be characterized by the ratio $\epsilon^{-1}=L / H_{x}$ (Fig. 11). Then, a question arises: can we relate the ratio $\epsilon^{-1}$ to the Froude number Fr? Fig. 12 shows the ratio $\epsilon^{-1}$, which will be named "deposit lengthening coefficient", versus the Froude number. No correlation is found between these two dimensionless numbers. On the curve displayed in Fig. 12, we distinguished data for which $r$ is greater than $r_{c r i t}$ on the one hand from the data for which $r$ is smaller than $r_{c r i t}$ on the other hand. First, two distinct groups appear. The "deposit lengthening coefficient" ranges from 40 to 100 for both groups but with different values of the Froude number of the incoming flows: $F r \approx 5-7$ for $r<r_{\text {crit }}$ (group A in Fig. 12 ) and $F r \approx 7-9$ for $r>r_{\text {crit }}$ (group B in Fig. 12). Second, in each group, a same Froude number can give a very distinct avalanche extension (see, in Fig. 12, the points $A_{1}$ and $A_{2}$ on the one hand and the points $B_{1}$ and $B_{2}$ in the other hand). This result highlights that not taking into account an internal property of the flowing material (the granulometry here) and the induced mechanism (the segregation here) can lead to an apparent macroscopic behavior being not intuitive. It points at a crucial implication for dry snow avalanches. Indeed, when operating avalanche zoning or designing a protection dam in avalanche engineering, it is necessary to calculate the maximum avalanche extension on the basis of average models: either the simplified analytical models (see, for example, the method proposed by (Salm and others, 1990) and largely used in avalanche engineering) or the advanced numerical models based on 
shallow depth-averaged equations (for example (Naaim and others, 2004)). These models are based on the basal effective friction coefficient, $\mu$, which has been to be estimated as a function of the snow type in particular. The friction coefficient $\mu$ drives the runout distance when using these analytical or numerical models. Given that the small-scale experiments presented in this paper show the strong influence of the granulometry on the avalanche runout (a same Froude number but a different $r$ can lead to two different values of $\epsilon), \mu$ should be chosen by taking into account the snow granulometry. Future research should be devoted to the determination of the friction coefficient as a function of the proportion in small snow grains and the mean diameter of the large aggregates, the ratio between the larger aggregates and the smaller grains, and also the particle shapes.

\subsection{Influence of the diameters ratio and size effect}

Our study at small-scale shows that segregation induces an increased mobility if the fine particles proportion is at a critical value $(0.25$ in our experiments). The ratio $D=d_{2} / d_{1}$ was fixed between $4\left(d_{1}^{\min }=250 \mu \mathrm{m}\right)$ and $6.66\left(d_{1}^{\max }=150 \mu \mathrm{m}\right)$ in our tests. Linares-Guerrero and others (2007) have analysed the effect of this ratio. They have shown that the higher the difference between the diameter of large and small particles, the more important the effect of segregation is. We have to keep in mind that all the effects observed would have been amplified if we would have had a larger $D$, and conversely, softened if we would have had a smaller $D$. The mobility will be more and more enhanced when the ratio $D$ will be higher. We can see here a kind of size effect. Indeed, if the avalanche volume is larger, its flow depth is larger and the maximum size of snow aggregates may be 
also larger. The idea suggested here relies on the statement that the initial largest blocks and chunks can keep their size, or some of the blocks or chunks may grow, during the flow propagation leading to snow aggregates of large size. It is a challenge to investigate such a statement by well-targeted snow experiments in the future. However, if this statement is true, it may result in a large ratio between the typical size of snow aggregates and the size of small snow grains, and consequently amplified segregation effects. In this sense, we think that the segregation mechanism may be a relevant candidate to enhance the mobility of major dry and dense snow avalanches. It could provide arguments to explain the field measurements showing a decrease of the effective friction as a function of the volume generally observed for geophysical flows (Legros, 2002), and also for snow avalanches (Ancey, 2005). If we consider a snow avalanche made of snow aggregates of typically $10 \mathrm{~cm}$ in size $\left(d_{2}=0.1 \mathrm{~m}\right.$ (Bartelt and McArdell, 2009)) and millimetric snow grains $\left(d_{1}=10^{-3} \mathrm{~m}\right)$, then the ratio $D$ can reach a value extremely high equal to 100 ! It would result a very low value of the effective friction coefficient if the proportion in fine particles is close to the value corresponding to a minimum (for $r=r_{c r i t}$ ). Coming back to our small-scale experiments, the critical proportion is $r_{\text {crit }}=0.25$ for $4<D<6.66$. From the preliminary results of Linares-Guerrero and others (2007, figure 12), the ratio $r_{\text {crit }}$ is likely to decrease when $D$ increases. Thus, it can be an additional amplication factor. At high $D$ values, a weak proportion of fine particles (small $r_{c r i t}$ ) is sufficient to produce a largely enhanced mobility. In parallel to researches already mentioned in conclusion of the previous section, it appears crucial to systematically investigate the granulometry of snow avalanches deposits 
when full-scale avalanches are surveyed following the example of Issler and others (2008). A recent study showed that the granular-size distributions in avalanche snow deposits is nearly log-normal (Bartelt and McArdell, 2009). Further investigations on polydisperse granular materials are also a future challenge to corroborate or not the conclusions reported here for simple binary granular mixtures. The segregation mechanism can also take place in many other geophysical flows where the flowing material is polydisperse with the presence of fine particles, with a large difference in diameter between fine and large particles, and with various shape particles.

\section{Acknowledgments}

The authors acknowledge financial support from the French National Research Agency (ANR-MONHA). The authors thank Mohamed Naaim for very fruitful discussions, and T. Faug is grateful to C. Schlosser for her help to initiate the preliminary experiments on bidisperse granular avalanches. We would like to thank to an anonymous reviewer for the constructive criticisms which helped to improve the quality of the paper.

\section{References}

Ancey, C. 2005. Monte carlo calibration of avalanches described as coulomb fluid flows. Phil. Trans. R. Soc. of London, 363, 1529-1550.

Bartelt, P. and B.W. McArdell. 2009. Instruments and Methods. Granulometric investigations of snow avalanches. J. Glaciol., 55, 829-833.

Ben Aim, R. 1970. Etude de la texture des empilements de grains. Application à la détermination de la perméabilité des mélanges binaires en régime 
moléculaire, intermédiaire, laminaire. PhD thesis, Faculté des Sciences de Nancy.

Berton, G., R. Delannay, P. Richard, N. Taberlet, and A. Valance. 2003. Two-dimensional inclined chute flows : Transverse motion and segregation. Physical Review E, 68, 051303.

Cochard, S. and C. Ancey. 2008. Tracking the free surface of time-dependent flows: Image processing for the dam-break problem. Experiments in Fluids, 44, 59-71.

Dodds, J. A. 1980. The porosity and contact points in multicomponent random sphere packings calculated by a simple statistical geometric model. Journal of Colloid and Interface sciences, 77 (2), 317-327.

Faug, T., P. Gauer, K. Lied, and M. Naaim. 2008. Overrun length of avalanches overtopping catching dams: Cross-comparison of small-scale laboratory experiments and observations from full-scale avalanches. $J$. Geophys. Res., 113, F03009.

Faug, T., M. Naaim, D. Bertrand, P. Lachamp, and F. Naaim-Bouvet. 2003. Varying dam height to shorten the run-out of dense avalanche flows: Developing a scaling law from laboratory experiments. Surv. Geophys., 24 (5-6), 555-568.

Faug, T., M. Naaim, and F. Naaim-Bouvet. 2004. An equation for spreading length, center of mass and maximum run-out shortenings of dense avalanche flows by vertical obstacles. Cold Reg. Sci. Technol., 39, 141151. 
Félix, G. and N. Thomas. 2004. Evidence of two effects in the size segregation process in dry granular media. Physical Review, 70, 051307-1-16.

Forterre, Y. and O. Pouliquen. 2008. Dense granular flows. Ann. Rev. Fluid Mech., 40, 1-24.

GDR-MiDi, . 2004. On dense granular flows. E. Phys. J. E, 14, 367-371.

Goujon, C., B. Dalloz-Dubrujeaud, and N. Thomas. 2007. Bi-disperse granular avalanche on inclined planes: a rich variety of behaviors. Eur. Phys. J. E., 23, 199-215.

Goujon, C., N. Thomas, and B. Dalloz-Dubrujeaud. 2003. Monodisperse dry granular flows on inclined planes: Role of roughness. Eur. Phys. J. E., 11, $147-157$.

Gray, J M N T. and V A. Chugunov. 2006. Particle-size segregation and diffusive remixing in shallow granular avalanches. J. Fluid Mech., 569, 365-398.

Hákonardóttir, K M., A.J. Hogg, T. Jóhannesson, and G.C. Tómasson. 2003. A laboratory study of the retarding effects of braking mounds on snow avalanches,. J. Glaciol., 49, 191-200.

Hirshfeld, D. and D C. Rapaport. 1997. Molecular dynamics studies of grain segregation in sheared flow. Physical Review E, 56(2), 20122018.

Hutter, K. 1996. Avalanche dynamics. Hydrology of disasters. Dordrecht, Boston, London: Kluwer Academic Publisher, chapter 11, pages 317-394. 
Hutter, K., T. Koch, C. Pluss, and S B. Savage. 1995. The dynamics of avalanches of granular-materials from initiation to runout. 2. experiments. Acta Mechanica, 109 (1-4), 127-165.

Issler, D. 2003. Experimental information on the dynamics of dry-snow avalanches. Dynamic response of granular and porous materials under large and catastrophic deformation, Springer, Berlin, pages 109-160.

Issler, D., A. Errera, S. Priano, H. Gubler anb B. Teufen, and B. Krummenacher. 2008. Inferences on flow mechanisms from snow avalanche deposits. A. Glaciol., 49, 187-192.

Legros, F. 2002. The mobility of long-runout landslides. Eng. Geol., 63, $301-331$.

Linares-Guerrero, E., C. Goujon, and R. Zenit. 2007. Increased mobility of bidisperse granular avalanches. J. Fluid Mech., 593, 475-504.

Naaim, M., F. Naaim-Bouvet, T. Faug, and A. Bouchet. 2004. Dense snow avalanche modeling: flow, erosion, deposition and obstacle effects. Cold Reg. Sci. Technol., 39 (2/3), 193-204.

Ottino, J M. and D V. Khakhar. 2000. Mixing and segregation of granular materials. Annu. Rev. Fluid Mech., 32, 55-91.

Phillips, J C., A J. Hogg, R R. Kerswell, and N H. Thomas. 2006. Enhanced mobility of granular mixtures of fine and coarse particles. Earth Plan. Sci. Lett., 246 (3-4), 466-480. 
Pouliquen, O., J. Delour, and S B. Savage. 1997. Fingering in granular chute flows. Nature, 386, 816-817.

Pouliquen, O. and Y. Forterre. 2002. Friction law for dense granular flows: application to the motion of a mass down a rough inclined plane. J. Fluid Mech., 453, 133-151.

Pouliquen, O. and J W. Vallance. 1999. Segregation induced instabilities of granular fronts. Chaos, 9, 621-630.

Pudasaini, S.P and K. Hutter. 2007. Avalanche Dynamics. Dynamics of Rapid Flows of Dense Granular Avalanches. Springer-Verlag.

Rognon, P., J N. Roux, M. Naaim, and F. Chevoir. 2007. Dense flows of bidisperse assemblies of disks down an inclined plane. Physics of Fluids, 19, 058101.

Rognon, P G., F. Chevoir, H. Bellot, F. Ousset, M. Naaim, and P. Coussot. 2008. Rheology of dense snow flows: Inferences from steady state chuteflow experiments. J. Rheol., 52 (3), 729-748.

Salm, B., A. Burkard, and H U. Gubler. 1990. Berechnung von fliesslawinen: Eine anleitung fuer praktiker mit beispielen. Technical report. Mitt. 47, Eidg. Inst. Schnee- und Lawinenforsch., Davos, Switzerland.

Sansoni, G., M. Carocci, and R. Rodella. 1999. Three-dimensional vision based on a combination of grey-code and phase-shift light projection: analysis and compensation of systematic errors. Applied Optics, Optical Society of America, 38 (31), 6565-6573. 
Savage, S B. 1979. Gravity flow of cohesionless granular materials in chutes and channels. J. Fluid Mech., 92, 53-96.

Savage, S B. and K. Hutter. 1989. The motion of finite mass of granular material down rough incline. J. Fluid Mech., 199, 177-215.

Savage, S B. and C K K. Lun. 1988. Particle size segregation in inclined chute flow of dry cohesionless granular solids. J. Fluid Mech., 189, 311-335.

Staron, L. and E. Lajeunesse. 2009. Understanding how volume influence the mobility of dry debris flows? Geophys. Res. Lett., 36, L12402.

Surrel, Y. 2000. Fringe analysis. Photomechanics, Topics Appli. Phys., 77, $55-102$.

Tai, Y C., J M N T. Gray, K. Hutter, and S. Noelle. 2001. Flow of dense avalanches past obstructions. A. Glaciol., 32, 281-284.

Vincent, C. 2003. Recalage et fusion pour reconstruire des surfaces 3D obtenues par projection de franges. Application à un système de vision dédié à l'orthopédie. PhD thesis, Université Jean Monnet de Saint-Etienne.

Zanuttigh, B. and A. DiPaolo. 2006. Experimental analysis of the segregation of dry avalanches and implications for debris flows. J. Hydrau. Res., 44 (6), 796-806. 


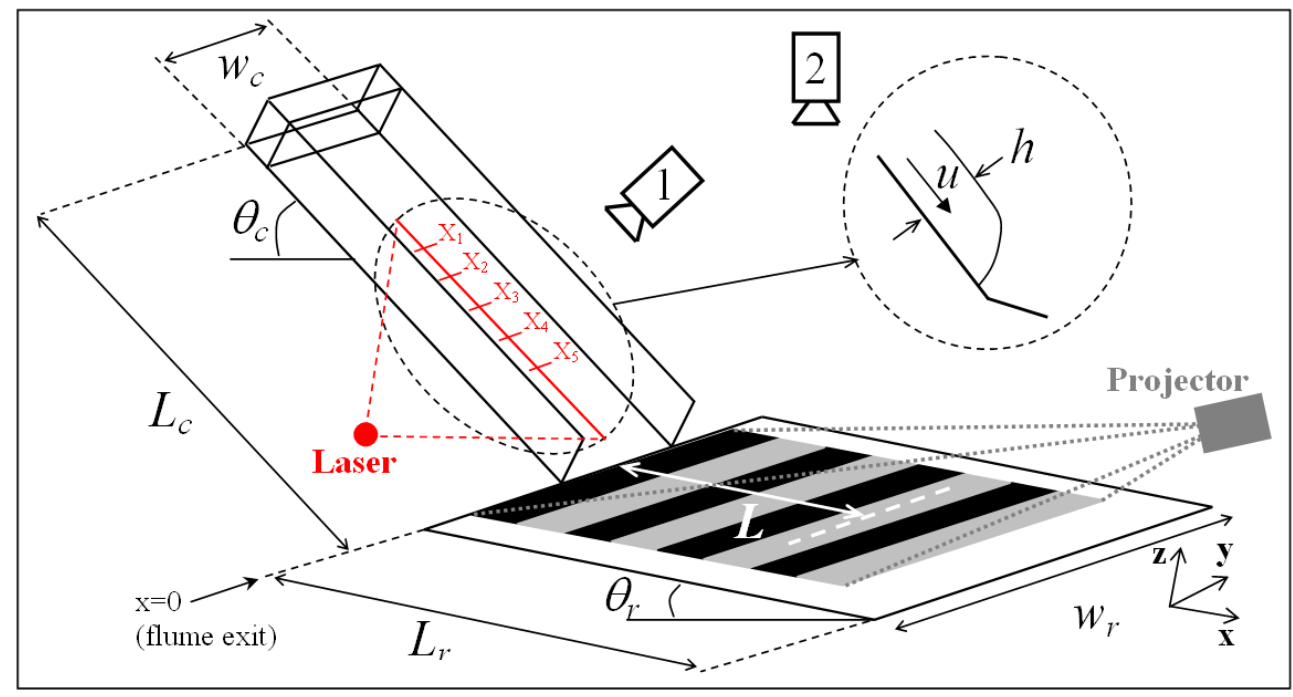

Figure 1: Sketch of the experimental set-up and instrumentation. The channel width was $w_{c}=0.30 \mathrm{~m}$, its length was $L_{c}=1.50 \mathrm{~m}$ and its slope inclination was fixed at $\theta_{c}=35^{\circ}$. A laser sheet was projected on the channel bottom to visualize, thanks to a digital camera $\left(\mathrm{n}^{\circ} 1\right)$, the displacement of the granular mass (flow depth $h$ and front velocity $u$ ) in the flowing zone. The locations $x_{1}$ was at $55 \mathrm{~cm}$ from the reservoir exit and the distance between two successive positions $x_{i}$ was around $13.75 \mathrm{~cm}$. The width of the downstream plane was $w_{r}=1.20 \mathrm{~m}$, its length was $L_{r}=2.50 \mathrm{~m}$ and its slope inclination was $\theta_{r}=6^{\circ}$. Alternatively black and white fringes were projected on the bottom of the downstream plane to visualize, thanks to a digital camera $\left(n^{\circ} 2\right)$, the boundaries of the granular mass in the runout zone (see Figure 4). 


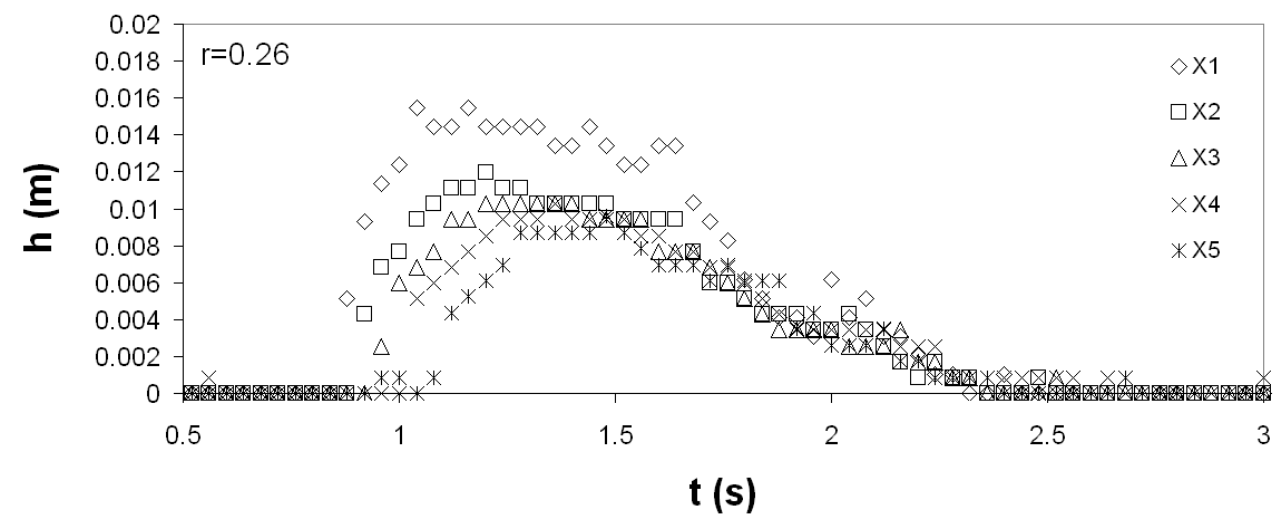

Figure 2: Flow-depth temporal profiles measured thanks to the shifted laser line at different channel locations. Example for $r=0.26$ : the height $h$ retained is the mean value of the maxima at locations $X_{3}\left[h\left(X_{3}\right)=10 \mathrm{~mm}\right], X_{4}\left[h\left(X_{4}\right)=9.3 \mathrm{~mm}\right]$ and $X_{5}\left[h\left(X_{5}\right)=8.8 \mathrm{~mm}\right]$, which gives $h \approx 9.37 \mathrm{~mm}$. 


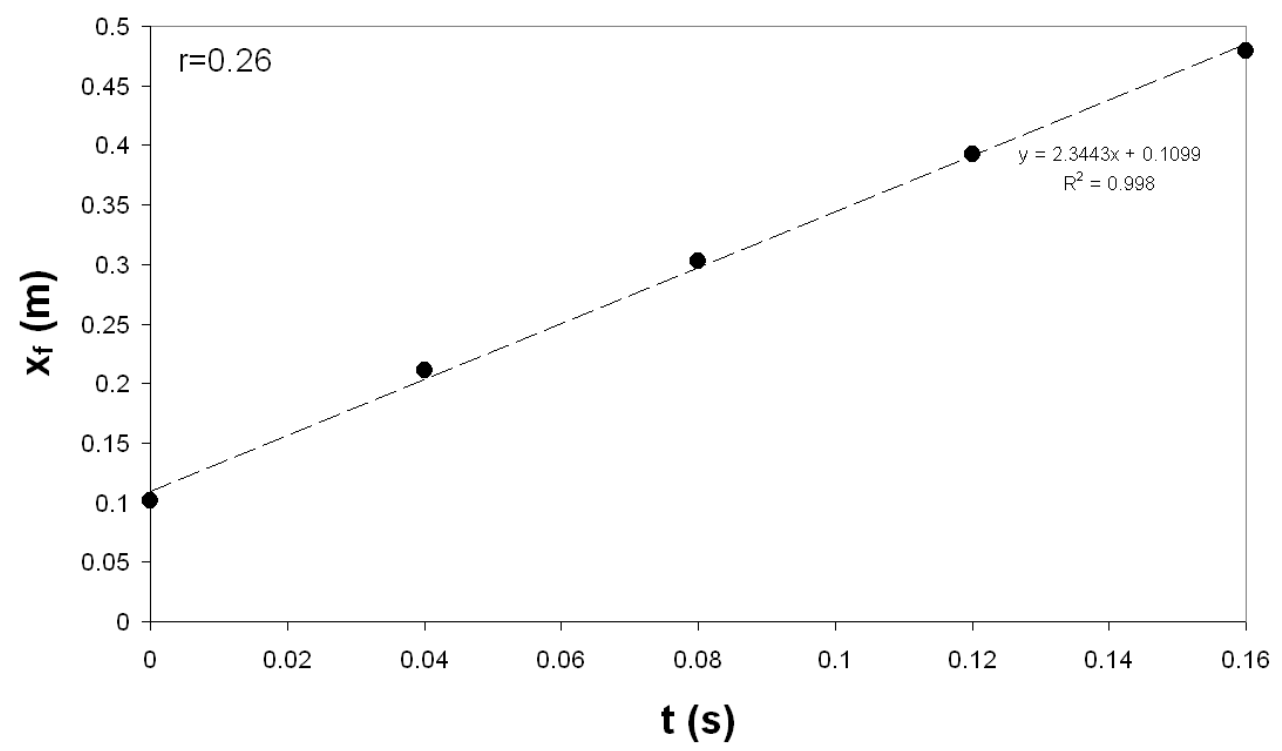

Figure 3: Granular front position $x_{f}$ in the channel versus the time. Example for $r=0.26$. The slope of the straight line allows us to estimate the mean front speed $(u)$ in the investigated flow zone where the granular mass deflects the laser line (see figure 1). 

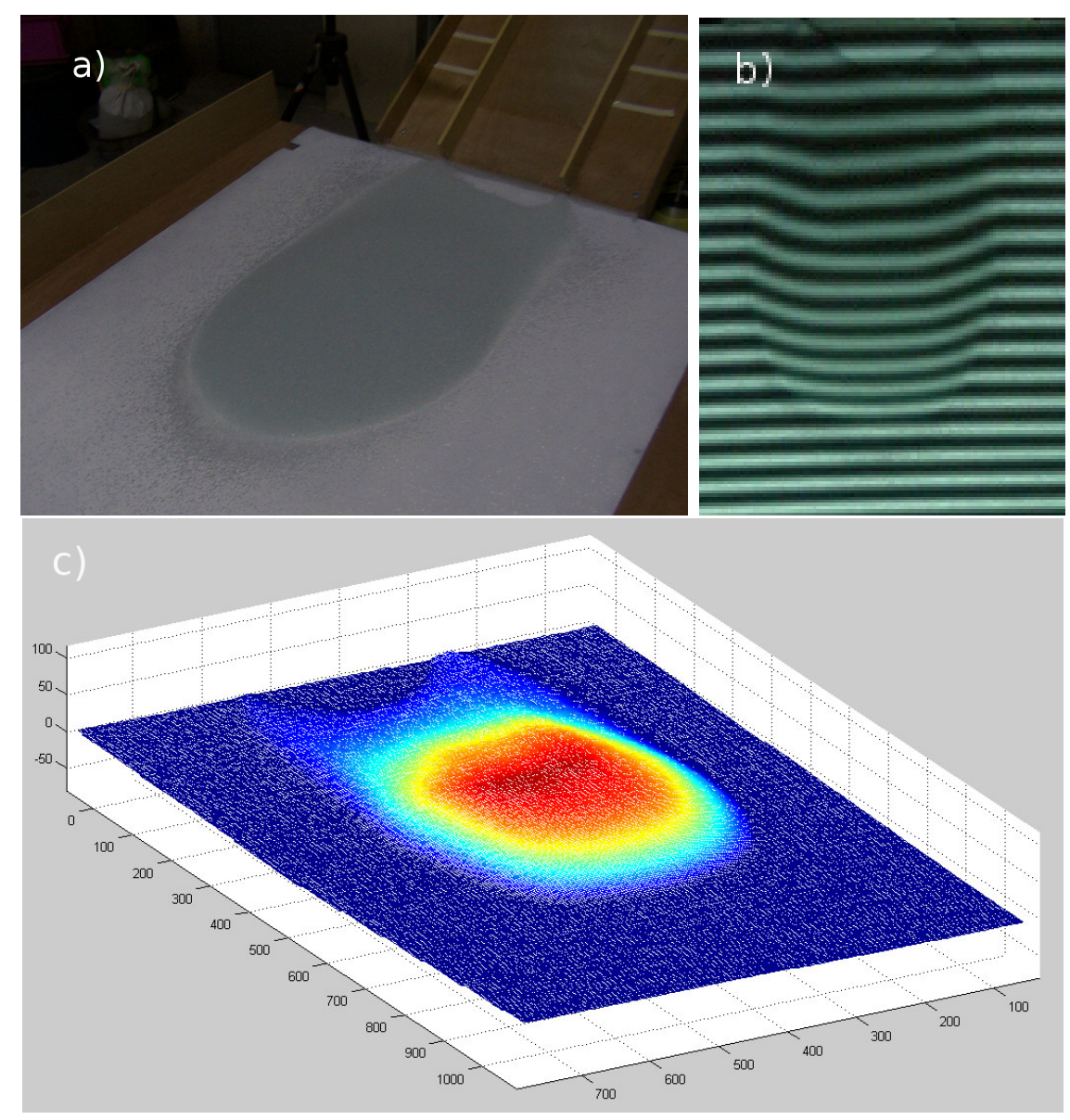

Figure 4: (a) Photograph of a granular deposit in the runout zone, (b) image of a fringes network projected on a deposit on the runout zone and (c) example of a numerical deposit obtained by the phase shifting method (color levels indicate the value of the deposit thickness, from the low heights in blue color to the larger ones in red color (the numerical values given on axis correspond to pixels). 


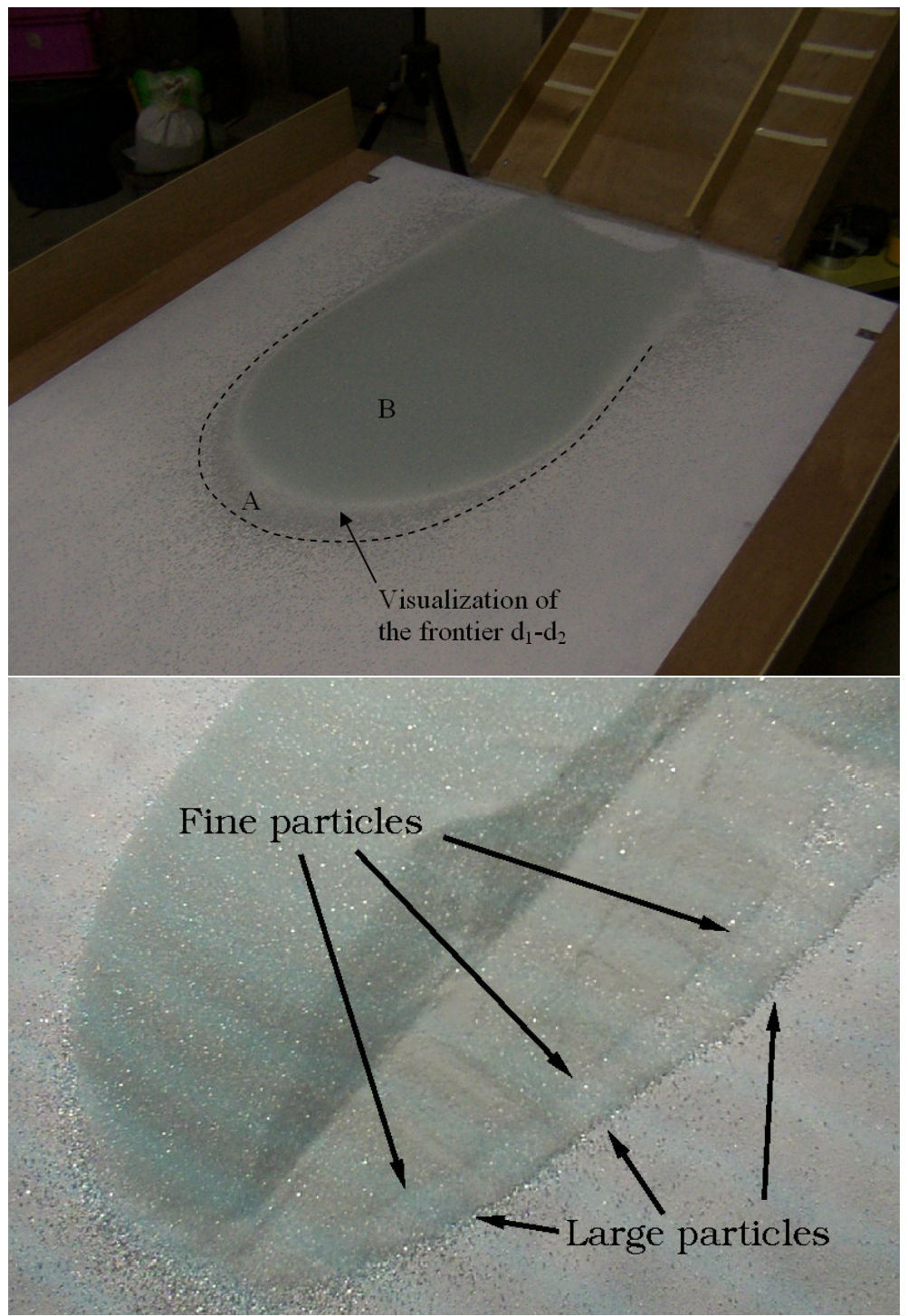

Figure 5: (top) Visualization of the frontier above which no fine particles are present. The area $\mathrm{A}$ is a location where only large particles of diameter $d_{2}=1 \mathrm{~mm}$ are present. The area $\mathrm{B}$ is a location where we can find both fine and large particles with a majority of fine particles, of diameter $d_{1}$, located at the base of the deposit. The frontier between the areas A and B is visible on the photograph and appears to be white colored, which provide evidence of the segregation operating inside the bidisperse granular material. (bottom) Some material has been removed from the deposit to show the evidence of segregation processes: a bed of fine particles is located 38 the base of the flow while the larger ones are above and go further (in the main flow direction and also laterally). 


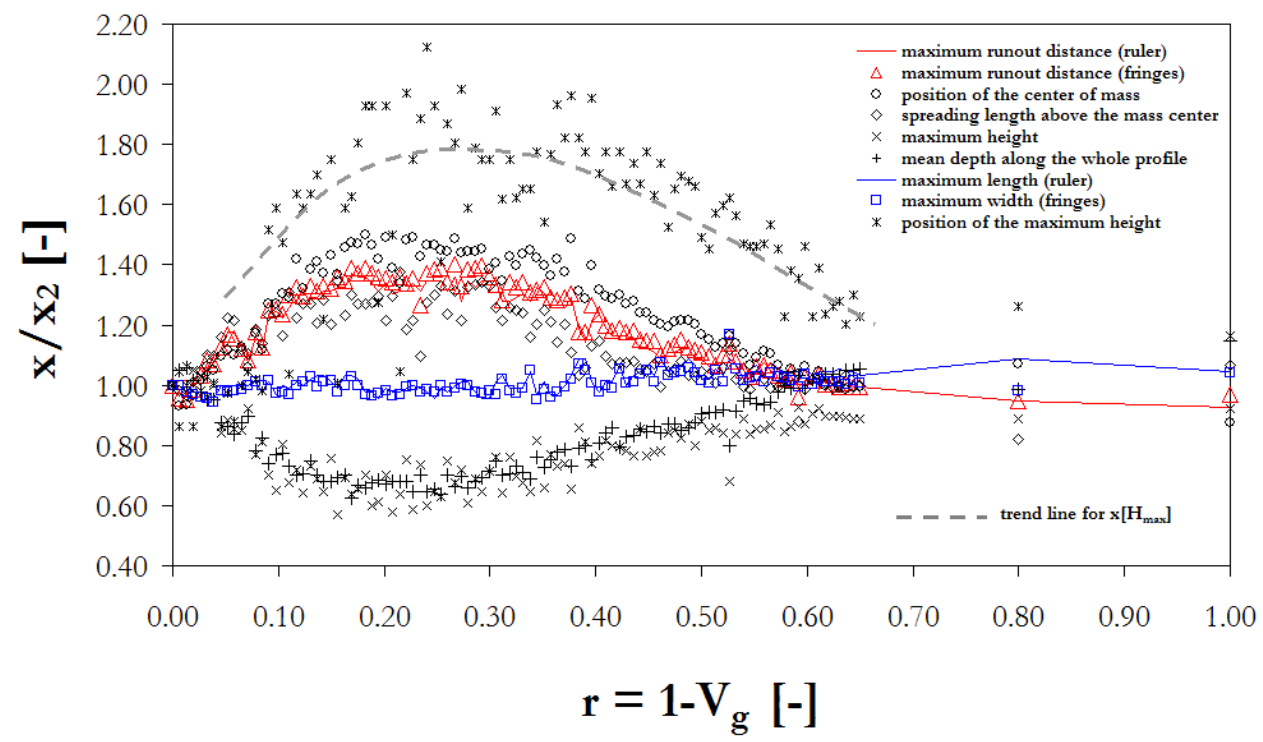

Figure 6: Various features of the granular avalanche deposit versus the mass proportion $r$ in fine particles: length $L$ (triangle symbols), position $x_{G}$ of the center of mass (circle symbols), spreading length $x_{\text {spreading }}$ above the center of mass (diamond symbols), maximum width $\ell$ (square symbol), maximum height $H_{\max }$ (times symbol), mean depth along the whole profile $H_{x}$ (cross symbols) and position $x\left[H_{\max }\right]$ of the maximum height (star symbols). Each dimension, $x$, has been normalized by the corresponding dimension, $x_{2}$, obtained with the monodisperse mixture of beads with diameter $d_{2}=1 \mathrm{~mm}(r=0)$. Note that the results from the direct measurements with a ruler are also shown for $L / L_{2}$ (red line) and $\ell / \ell_{2}$ (blue line) to validate the fringes projection technique. Since large and fine particles have the same density, the mass ratio $r$ is equal to $1-V_{g}$, where $V_{g}$ is the volume proportion of large grains. 


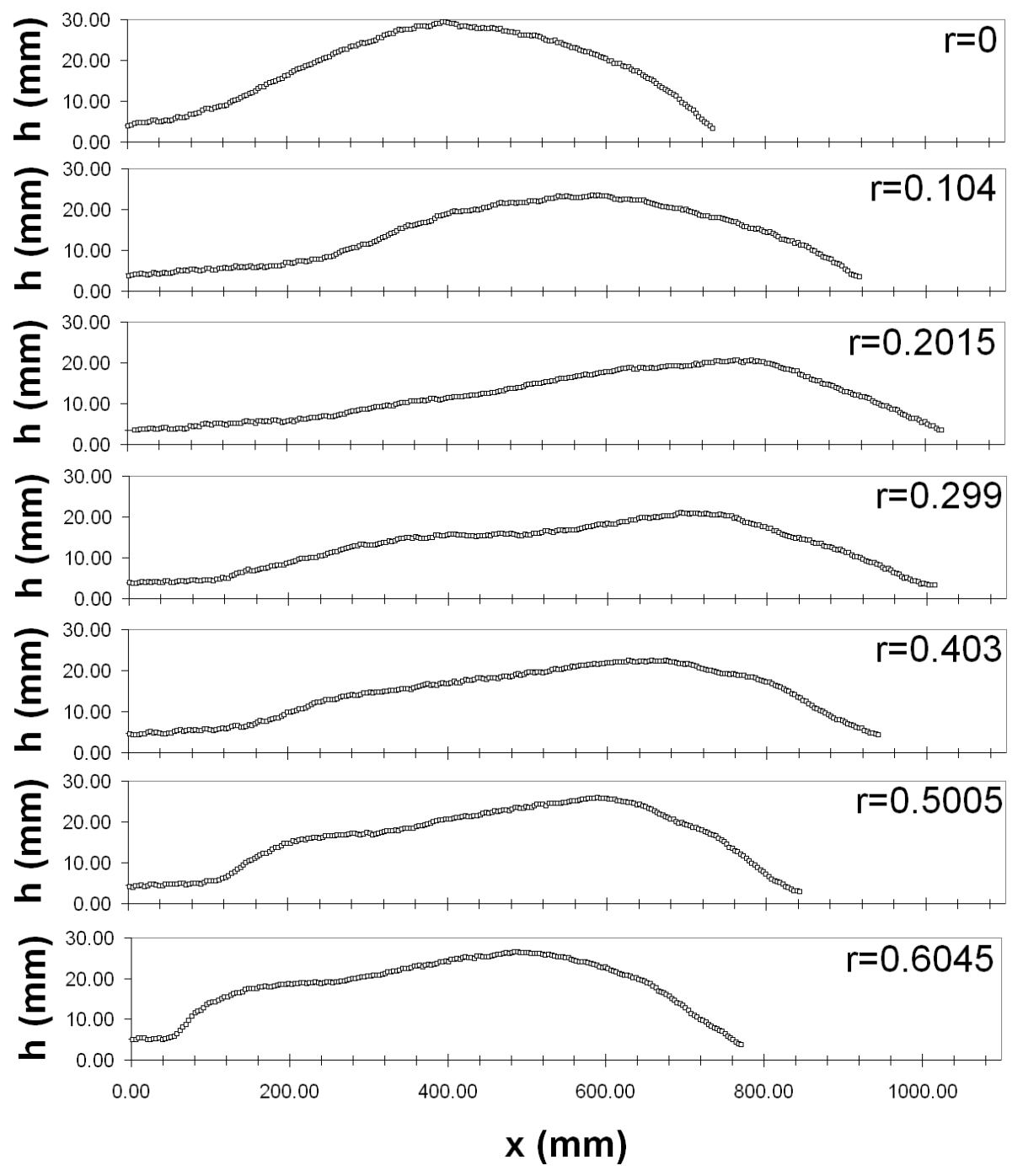

Figure 7: Evolution of the deposit shape as a function of the mass proportion, $r$, in fine particles: mean depth profile $(H)$ for various mass proportions in fine particles: $r=0$, $r=0.104, r=0.2015, r=0.299, r=0.403, r=0.5005, r=0.6045$ (see Figure 1 for the definition of $x=0$ corresponding to the flume exit). 


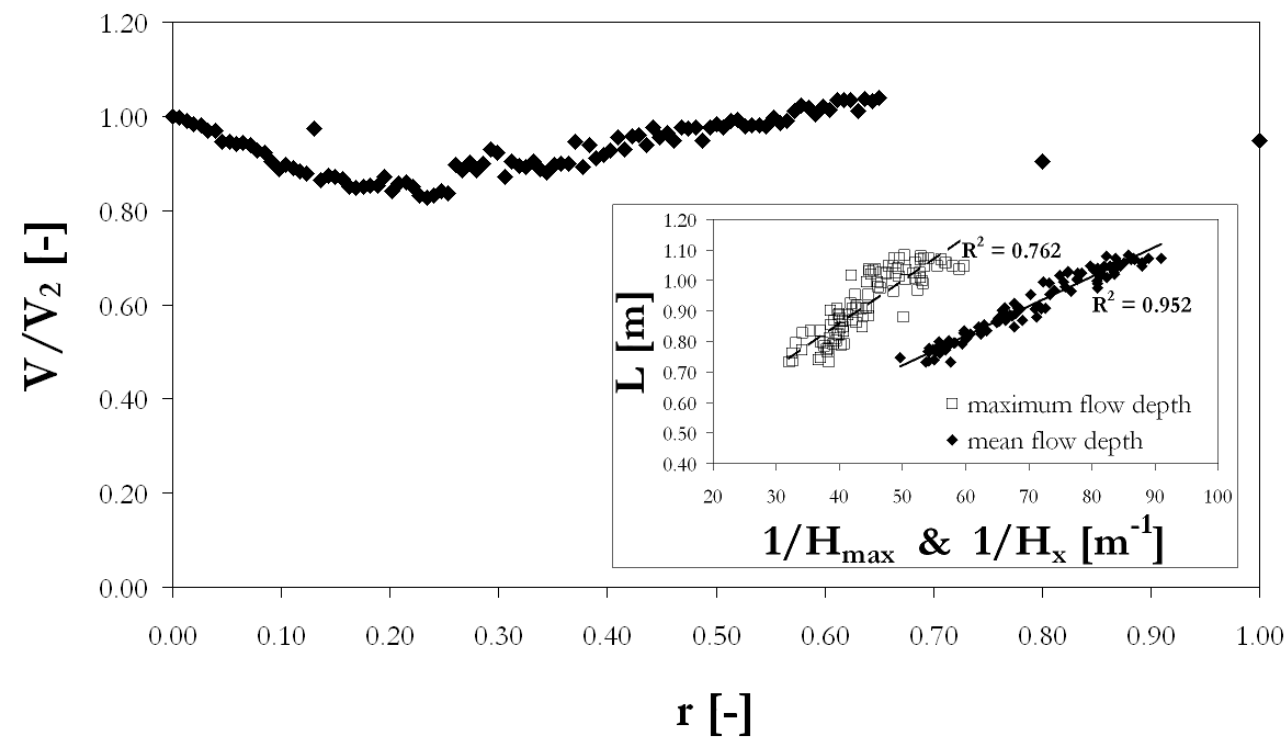

Figure 8: Normalized final volume of the deposit, $V / V_{2}$, versus the mass proportion $r$ in fine particles. The laboratory tests were carried out at constant initial released volume $(m=10 \mathrm{~kg})$, which results in a solid fraction variation conversely proportional to the volume variation. Insert: Length of the deposit, $L$, versus the inverse of the mean flow depth all along the profile, $1 / H_{x}$, and the inverse of the maximum deposit height, $1 / H_{\max }$. 


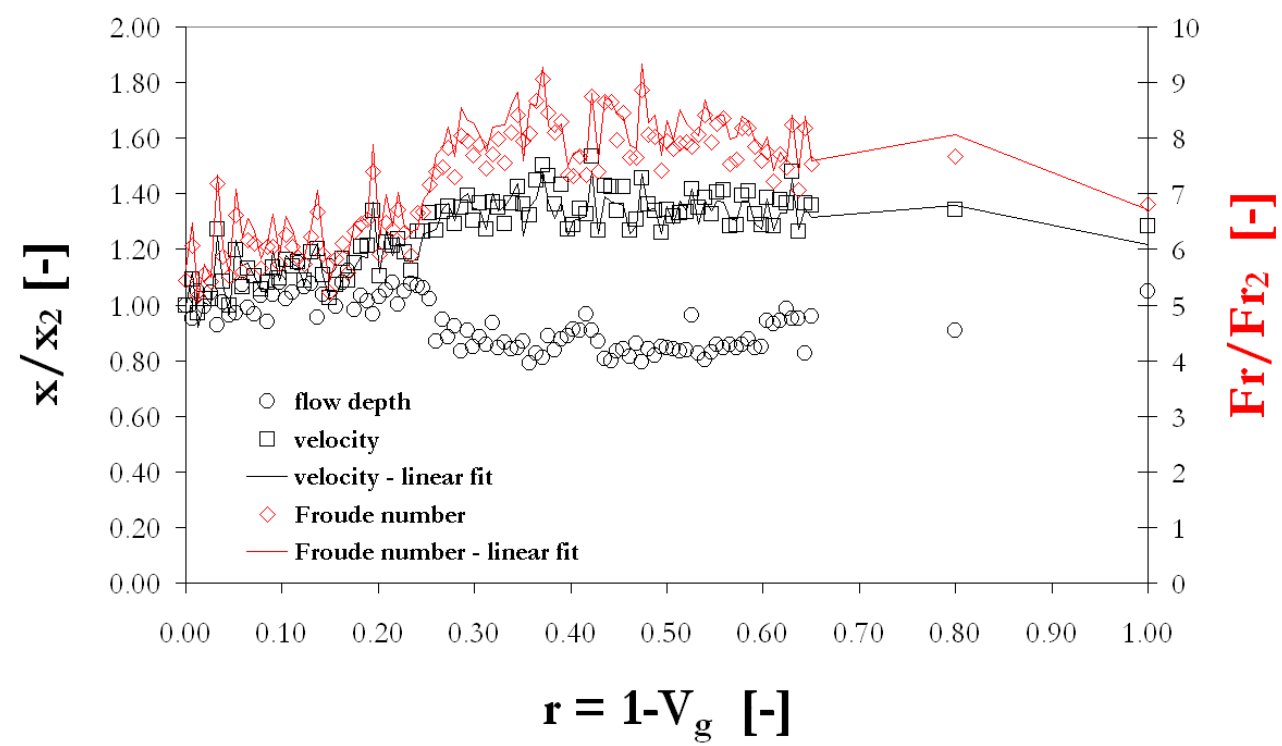

Figure 9: Various dynamic features of the incident channelized flow versus the mass proportion $r$ in fine particles: average flow depth $h$ (circle symbols), average avalanche front velocity $u$ (square symbols) and avalanche Froude number $\operatorname{Fr}$ (diamond symbols). $h, u$ and $F r$ have been normalized by the corresponding value $x_{2}$ obtained in presence of large grains only $(r=0)$. Since large and fine particles have the same density, the mass ratio $r$ is equal to $1-V_{g}$, where $V_{g}$ is the volume proportion of large grains. 


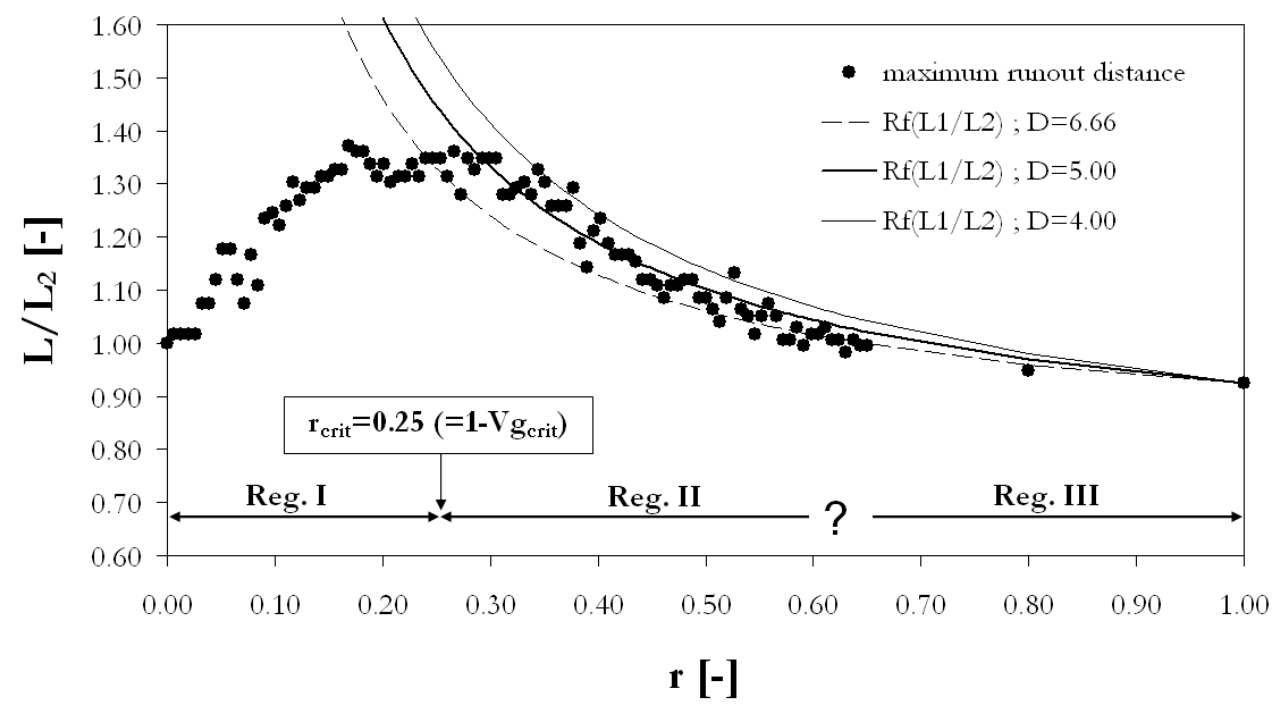

Figure 10: Length, $L$, of the deposit normalized by the length, $L_{2}$, of the deposit obtained with the large grains of diameter $d_{2}$ only $(r=0)$ versus the mass proportion $r$ in fine particles: definition of the mobility regimes (I), (II) and (III) and comparison of the data to the heuristic model for the regime (III). The transition between the regimes (III) and (II) is around $r=0.7$. From their experiments, Philipps and others (2006) proposed also a transition around $r^{I I-I I}=0.7$. We reported the model predictions, i.e. the value of $R_{f}\left(L_{1} / L_{2}\right)$, for $D=4, D=5$ and $D=6.66$. 


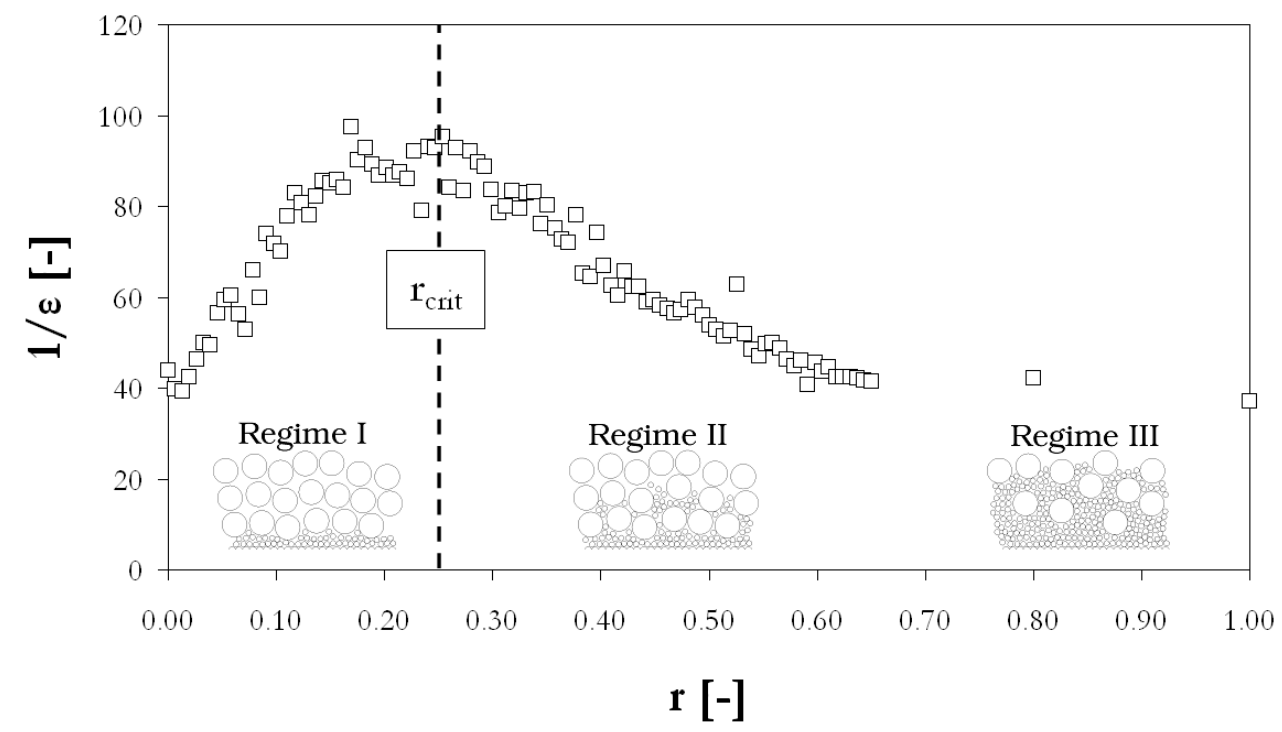

Figure 11: Ratio of the deposit length to the mean deposit height, $\epsilon^{-1}=L / H_{x}$, versus the mass proportion $r$ in fine particles of diameter $d_{1}$. 


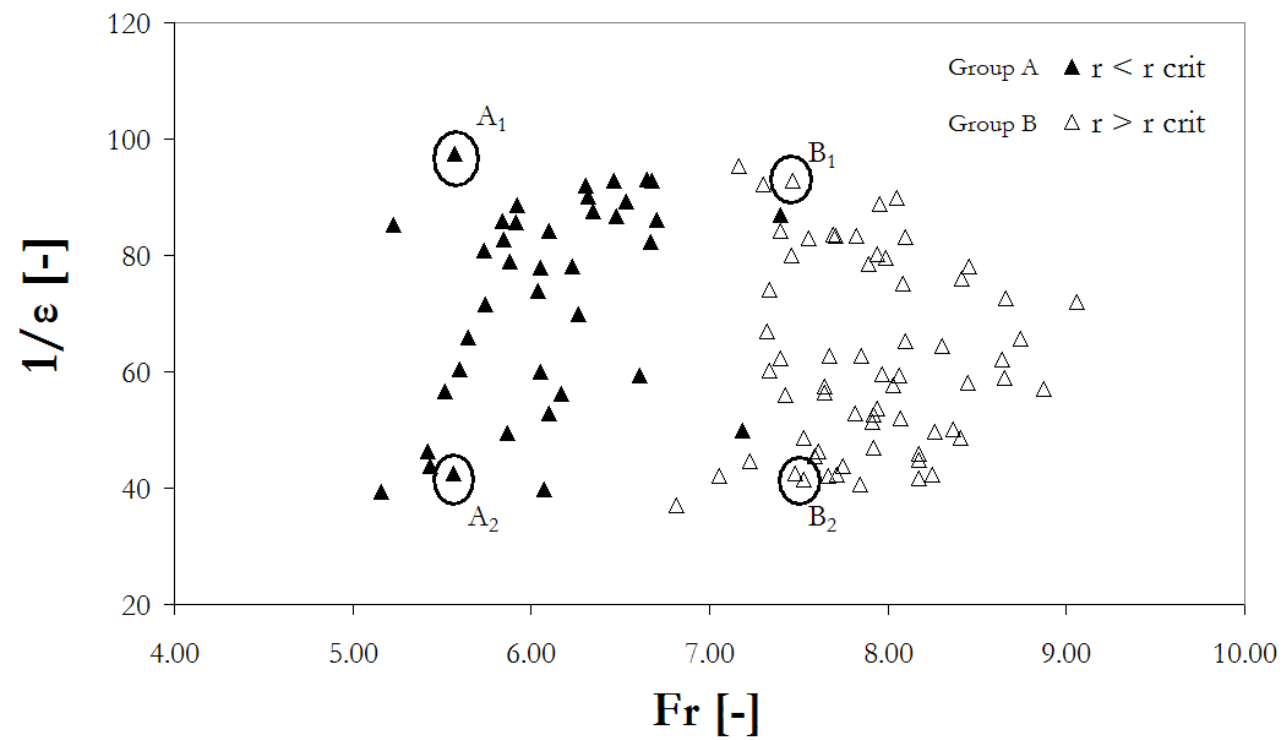

Figure 12: Lengthening coefficient of the deposit in the runout zone, $\epsilon^{-1}=L / H_{x}$, versus the Froude number $F r$. The critical value of the ratio $r$ is about $r_{c r i t}=0.25$ in our experiments. This value depends on the value of the ratio $D=d_{2} / d_{1}$ according to the discrete numerical simulations carried out by Linares-Guerrero and others (2007). 